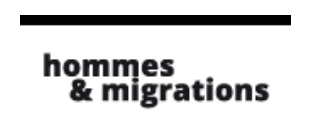

Hommes \& migrations

Revue française de référence sur les dynamiques

migratoires

$1298 \mid 2012$

France - Algérie, le temps du renouveau

\title{
Développer l'entrepreneuriat entre les deux rives
}

Entretien avec Fetah Ouazzani, président du Réseau des Algériens diplômés des grandes écoles et universités françaises (REAGE).

\section{Marie Poinsot}

\section{(2) OpenEdition Journals}

Édition électronique

URL : http://journals.openedition.org/hommesmigrations/1874

DOI : 10.4000/hommesmigrations. 1874

ISSN : 2262-3353

Éditeur

Musée national de l'histoire de l'immigration

Édition imprimée

Date de publication : 1 juillet 2012

Pagination : $38-41$

ISSN : 1142-852X

Référence électronique

Marie Poinsot, «Développer l'entrepreneuriat entre les deux rives », Hommes \& migrations [En ligne], 1298 | 2012, mis en ligne le 01 juillet 2014, consulté le 20 avril 2019. URL : http://

journals.openedition.org/hommesmigrations/1874; DOI : 10.4000/hommesmigrations.1874 


\title{
Développer l'entrepreneuriat entre les deux rives
}

\author{
Entretien avec Fetah Ouazzani, \\ président du Réseau des Algériens diplômés des grandes écoles \\ et universités françaises (REAGE), réalisé par Marie Poinsot
}

\section{Hommes \& Migrations : L'immigration algérienne en France est-elle selon vous un atout pour le développement des échanges avec l'Algérie?}

Fetah Ouzzani : Plus qu'un atout, l'immigration algérienne en France est un véritable levier stratégique, que ce soit pour les échanges commerciaux, le transfert des technologies et des savoirs ou le développement des partenariats industriels. Chaque entrepreneur, chaque enseignant ou chaque étudiant en Algérie a ses relais dans la communauté algérienne de France qui est constituée de plus de 3 millions de personnes, avec en son sein plus de 300000 cadres et chefs d'entreprise. De véritables réseaux se sont constitués ces vingt ou trente dernières années (commerce, transfert d'argent, etc.). On peut citer deux indicateurs pour rendre compte de cette situation : le nombre de villes en Algérie desservies par Air Algérie et Aigle Azur à partir de la France et le nombre d'étudiants algériens en France (23000 en 2012 d'après l'ambassade de France en Algérie), sachant que l'immense majorité restera en France définitivement. Le retour au pays reste en général un vceu pieux...

\section{H\&M : Quel est l'apport des migrants et de leurs enfants au dévelop- pement économique de l'Algérie ?}

F. O. : On peut classer les apports selon trois grandes périodes. D’abord, celle des années cinquante jusqu'aux années quatre-vingt où les immigrés, en majorité des ouvriers et des techniciens, subvenaient aux besoins de leurs familles restées au bled en envoyant des mandats chaque mois. C'était le principal apport. Ensuite, pendant la petite période de la décennie noire (les années quatre-vingt-dix), des réseaux de solidarité se sont multipliés en faveur des familles au bled touchées par la tragédie qu'a vécue l'Algérie. Enfin, la troisième période, la plus significative, à partir des années deux mille et qui court toujours pendant laquelle une véritable classe moyenne d'origine algérienne s'est développée en France. Elle est constituée, 
d'une part, des descendants de l'immigration ouvrière historique (deuxième, troisième et quatrième générations) et, d'autre part, des cadres ayant étudié en France et qui, ensuite, s'y sont définitivement établis, en plus des milliers de cadres qui se sont réfugiés en France durant la décennie noire. Les apports de cette période sont multiples : investissement dans l'immobilier, dans des entreprises mixtes, dans des entreprises locales... Les principaux secteurs qui en bénéficient sont le commerce, l'hôtellerie-restauration, les TIC, l'agroalimentaire, la franchise, l'importation et l'immobilier. En termes de résultats, on peut parler de l'amélioration en Algérie de la qualité des produits et des services, et probablement de 25 à $30 \%$ du PIB (estimation basée sur les indicateurs économiques algériens)!

\section{H\&M : Les jeunes d'origine algérienne en France sont-ils motivés par des carrières en lien avec le pays d'origine de leurs parents ? Pourquoi ? Ont-ils la formation adéquate pour être des acteurs per- formants ?}

F. O. : Il est de notoriété publique que très peu de jeunes formés en France, notamment les "hauts potentiels", sont motivés par une carrière en Algérie. De nombreuses opportunités s'offrent à eux sur le marché international. Dans le même temps, 
des obstacles obstruent la voie algérienne : le coût de la vie et la faiblesse des salaires, la faible attractivité des entreprises publiques et le manque d'entreprises privées de rang international... en plus de la bureaucratie régnante. Néanmoins, depuis quelques années, on observe un regain d'intérêt pour le pays d'origine chez les cadres expérimentés, en raison, notamment, de la conjonction des opportunités offertes par un pays en émergence et du manque de perspectives en France en raison d'une crise économique persistante. De plus en plus de cadres supérieurs soutenus par leurs familles et leurs réseaux en Algérie créent leurs propres entreprises ou des filiales d'entreprises françaises en Algérie. D'autres sont recrutés directement par les multinationales qui opèrent en Algérie et visent à "algérianiser" le management. Ils arrivent "par le haut" dans les directions générales et bénéficient de package d'expatriés. En général, ces heureux élus sont formés dans les universités les plus réputées et dans les grandes écoles françaises dans les domaines clés de la nouvelle économie (industrie, énergie, services, distribution, agriculture, etc.). Parfois, on trouve des jeunes peu expérimentés qui acceptent de passer quelques années en Algérie dans des multinationales dans l'espoir de progresser plus vite qu'en France et de bénéficier ainsi d'une mobilité à l'international au bout de quelques années en Algérie. On les trouve essentiellement dans les métiers du conseil et des TIC.

H\&M : Quels sont les freins actuels à l'essor de ces échanges ? Le climat des relations diplomatiques entre les deux pays intervient-il ? Qu'en est-il de l'image des émigrés algériens auprès des acteurs économiques en Algérie et plus généralement de la culture professionnelle des jeunes (niveau de langue) qui souhaitent investir leurs compétences dans ce pays?

F. O. : La coopération économique entre les deux pays se maintient à un niveau appréciable et elle ne souffre pas trop du poids de l'histoire ni du phénomène de "yo-yo" de la relation politique. L'interdépendance des deux économies dans plusieurs domaines (énergie, santé, céréales, formation...) est un précieux "garde-fou" ! Cependant, le niveau de cette coopération est largement en deçà du potentiel disponible. On ne peut éluder quelques "vraies-fausses" difficultés (langue, culture...) qui ne constituent pas véritablement des freins. À mon sens, l'obstacle majeur se situe au niveau politique. La relation entre les deux pays n'est malheureusement pas encore assise sur une véritable vision stratégique partagée. Et pourtant, ces deux pays ont la légitimité et les moyens pour constituer rapidement ensemble un véritable socle en faveur d'une nouvelle économie eurafricaine qui, à terme, bénéficiera d'un marché de 1,3 milliard d'âmes ! En espérant qu'il ne sera pas trop tard tant les dragons asiatiques sont déjà sur le coup ! 


\section{H\&M : Quel est le rôle du REAGE pour favoriser et appuyer ces dévelop- pements économiques ?}

F. O. : La mission du REAGE comprend essentiellement trois volets. Le REAGE souhaite porter à la connaissance du plus grand nombre les potentialités et les opportunités de l'économie algérienne. Il entend contribuer par le levier de l'économie et son corollaire, l'entreprenariat, à l'amélioration et à la densification de la relation bilatérale entre la France et l'Algérie. Enfin, il participe par le biais de projets à la construction de partenariats "gagnant-gagnant" entre les entrepreneurs des deux rives.

\section{H\&M : Quels projets précis le REAGE mène-t-il actuellement ?}

F. O. : Le REAGE aide des entreprises françaises opérant en Algérie et des entreprises algériennes de rang international à recruter des cadres à haut potentiel. Il joue un rôle de facilitateur et de médiateur en faveur des membres porteurs de projets de création de filiales ou de nouvelles entreprises en Algérie (démarches administratives, financement, accès au marché....). Enfin, le REAGE apporte un soutien aux entrepreneurs algériens qui souhaitent exporter ou se développer en Europe via la France. En 2013, nous espérons monter un fonds d'investissement pour financer des projets en Algérie. Notre forum annuel aura lieu probablement en Algérie l'année prochaine. Nous organisons le 11 octobre prochain à Paris une grande conférence sur l'émergence des écosystèmes en Algérie. 